\title{
Instrumentos que miden la comunicación enfermera-paciente
}

\section{Instruments measuring nurse-patient communication}

\section{Instrumentos que medem a comunicação enfermeira-paciente}

\author{
M. Gijón-Alvarado ${ }^{\mathrm{a} *}$, M.C. Müggenburg-RodríguezVigil ${ }^{\mathrm{b}}$ \\ ORCID: \\ a $0000-0002-7546-7081$ \\ b $0000-0002-0497-8685$ \\ Coordinación de Investigación, Escuela Nacional de Enfermería y Obstetricia, \\ Universidad Nacional Autónoma de México, Ciudad de México, México \\ Recibido: 16 mayo 2018 \\ Aceptado: 7 febrero 2019
}

\section{Resumen}

Introducción: Por la importancia que tiene la comunicación al ofrecer un cuidado de calidad, las enfermeras se han preocupado por la evaluación de la interacción con su paciente en el ejercicio de su práctica profesional.

Objetivo: Identificar instrumentos que midan la interacción enfermera paciente, por el valor que puede implicar para la evaluación de la calidad de la atención en la dimensión interpersonal.

Metodología: Se revisó la literatura y consultaron las bases de Scielo, Medline, Redalyc, BVS y, CINHAL. De acuerdo con el propósito enunciado se seleccionaron 25 artículos que investigan sobre el diseño, validación, aplicación y adaptación de instrumentos que miden la comunicación enfermera-paciente en diversos servicios y niveles de salud.

Resultados: Los artículos seleccionados se clasificaron en tres grupos: a) aplicación y validación de instrumentos; b) adaptación y validación de instrumentos; c) diseño y validación de instrumentos. Nueve hacen referencia a la aplicación y validación de instrumentos, 10 tanto a la adaptación como la validación, por último, seis al diseño y validación.

Conclusiones: Aunque se han diseñado, validado, aplicado y ajustado instrumentos, aún existen retos en este campo de investigación, con el fin de ofrecer nuevas estrategias que diagnostiquen y brinden alternativas específicas, para mejorar la comunicación enfermera-paciente en la práctica diaria de enfermería. 
Palabras clave: Comunicación en salud; relación enfermera-paciente; cuidado de enfermería; psicometría-enfermería; México.

\begin{abstract}
Introduction: Given the importance which communication has in healthcare of quality, nurses have been concerned about the assessment of professional interactions with their patients.

Objective: To identify instruments measuring the nurse-patient interactions in order to assess the quality of attention provided within an interpersonal dimension.

Methodology: The related literature was reviewed by consulting the Scielo, Medline, Redalyc, BVS and CINHAL databases. 25 articles researching on the design, validation, application, and adaptation of instruments measuring nurse-patient communications in diverse services and levels of care were selected.

Results: Selected articles were classified into three groups: a) application and validation of instruments; b) adaptation and validation of instruments; c) design and validation of instruments. Nine articles make reference to the application and validation of instruments, 10 make it to adaptation and validation, and 6 make it to the design and validation.

Conclusions: Although diverse articles addressing the issue have been designed, validated, applied and adjusted, challenges in this research area still exist regarding new strategies which can diagnose and offer alternatives to improve the communication between the nurses and their patients in the daily nursing practice. Keywords: Health communication; nurse-patient relations; nursing care; psychometrics-nursing; Mexico.
\end{abstract}

\title{
Resumo
}

Introdução: Pela importância que tem a comunicação para oferecer um cuidado de qualidade, as enfermeiras têm se preocupado pela avaliação da interação com seu paciente no exercício de sua prática profissional.

Objetivo: Identificar instrumentos que medem a interação enfermeira paciente, pelo valor que pode envolver para a avaliação da qualidade da atenção na dimensão interpessoal.

Metodologia: Revisou-se a literatura e consultaram as bases de Scielo, Medline, Redalyc, BVS e, CINHAL. Conforme no propósito enunciado selecionaram-se 25 artigos que pesquisam sobre o desenho, validação, aplicação e adaptação de instrumentos que medem a comunicação enfermeira-paciente em diversos serviços e níveis de saúde.

Resultados: Os artigos selecionados classificaram-se em três grupos: a) aplicação e validação de instrumentos; b) adaptação e validação de instrumentos; c) desenho e validação de instrumentos. Nove fazem referência à aplicação e validação de instrumentos, 10 tanto na adaptação quanto na validação, por último, seis no desenho e validação.

Conclusões: Ainda que se tenham desenhado, validado, aplicado e ajustado instrumentos, ainda existem desafios neste campo de pesquisa com o fim de oferecer novas estratégias que diagnostiquem e forneçam alternativas específicas, para melhorar a comunicação enfermeira-paciente na prática diária de enfermagem.

Palavras chave: Comunicação em saúde; relações enfermeiro-paciente; cuidados de enfermagem; psicometria-enfermagem; México.

\section{Introducción}

La comunicación es una necesidad humana que forma parte de la vida cotidiana a lo largo del tiempo, se emplea como un proceso en que el emisor y el receptor pueden expresarse a través de signos, palabras y códigos, durante este proceso surge un intercambio de información por medio de conductas verbales y no verbales; en el caso de enfermería la comunicación entre este personal y el paciente es importante porque ocupa un lugar prioritario en el ejercicio de la práctica profesional, ya que es el eje articulador de los cuidados, tanto asistenciales, como tecnológicos, dirigidos a la oferta del cuidado de enfermería de calidad ${ }^{1}$. 
El personal de enfermería se comunica por ambos medios, lo que posibilita el establecimiento de una relación interpersonal significativa con el paciente cuando se imparte el cuidado. La percepción de señales corporales y de expresiones verbales representa la posibilidad de ir más allá de la ejecución de procedimientos técnicos al permitir que los sentimientos, las emociones y la historia de cada uno, favorezcan la comprensión integral del ser humano ${ }^{2,3}$.

La comunicación favorece el cuidado de calidad, requiere del establecimiento de una buena relación terapéutica y es una herramienta básica para sostener el proceso de cuidar, ya que mediante la comunicación con el paciente el profesional de enfermería puede planificar y brindar un cuidado más efectivo², ${ }^{4}$, que le permite establecer interacciones para la restauración de la salud del paciente, es importante aquello que se le transmite a este, no sólo con las palabras si no también con los gestos y expresiones, el comportamiento con los pacientes genera reacciones positivas o negativas que continuamente se pueden reencauzar en su beneficio.

Las habilidades de comunicación con el paciente son fundamentales para la práctica de los profesionales de enfermería y se constituyen como uno de los factores más importantes para la determinación de la calidad de los servicios de salud ${ }^{3}$. El fomento de elementos facilitadores de la relación terapéutica como la empatía, la escucha activa y el respeto a la dignidad del individuo favorece la comunicación eficaz y la relación terapéutica satisfactoria.

La empatía está vinculada con la habilidad de entender las experiencias y los sentimientos de otra persona, comprender a la otra persona desde su propio marco de referencia ${ }^{5}$. La empatía se asocia a atributos como el respeto y la escucha activa, ya que es necesario encontrarse en disposición física y mental para querer escuchar con atención a la persona, pero al mismo tiempo acogerla y recibirla sin emitir juicios de valor ${ }^{6,7}$.

La escucha activa es una técnica para comunicar mejor la aceptación y es un componente de la empatía, una de sus funciones consiste en ayudar a la persona a encontrar sus propias soluciones, a través de sus conocimientos, opiniones y creencias, para que se responsabilice y asuma sus decisiones ${ }^{3}$.

Por la importancia y la trascendencia que tiene la comunicación para ofrecer un cuidado de calidad, el personal de enfermería se ha preocupado por la evaluación de la interacción con su paciente durante el ejercicio de su práctica profesional. La valoración de la interacción con el paciente ha sido estudiada a través de metodologías cualitativas, cuantitativas y revisiones de la literatura.

En el primer caso varios autores se abocaron al análisis de la percepción de los cuidados en la UCI ${ }^{8,9}$ y a la evaluación de la comunicación en la unidad de urgencias ${ }^{2}$. Entre los artículos que emplearon metodologías cuantitativas se destacan los que se orientan a la valoración de la satisfacción de los pacientes con respecto al cuidado de enfermería ${ }^{10-12}$, la importancia del uso de las habilidades de comunicación ${ }^{7,13}$, la percepción de la calidad de los cuidados ${ }^{14,15}$, la valoración de la comunicación ${ }^{16}$ y el valor de la empatía en el área de enfermería ${ }^{6,17}$. En cuanto a las revisiones de la literatura se analizaron: el rol que ejerce el profesional de enfermería durante el proceso de comunicación con el paciente y el uso de las habilidades sociales en el área de enfermería ${ }^{4,18-20}$.

En el ámbito cuantitativo el personal de enfermería se ha ocupado de la medición de la comunicación interpersonal, han diseñado ${ }^{7,21-25}$, adaptado y validado ${ }^{6,10,16,26-35}$ o aplicado instrumentos ${ }^{12-15,36,37}$. Los resultados de las mediciones permiten identificar áreas de oportunidad para la mejora de la calidad de los servicios de enfermería y proponer estrategias para fortalecer la relación terapéutica.

La trascendencia de estas mediciones motiva a la búsqueda de instrumentos nacionales o internacionales, que permitan seleccionar aquellos que puedan ser utilizados para fortalecer la calidad de los cuidados de enfermería.

La aplicación de instrumentos de medición debe considerar elementos como: la validez y la confiabilidad, para que el uso de los resultados obtenidos durante la aplicación de éstos ofrezca información confiable, con el fin de sugerir las mejores estrategias para mejorar la práctica del cuidado. 
Dada la importancia de la medición de la comunicación enfermera-paciente, se propone la búsqueda y la revisión de instrumentos que midan esta comunicación, por el valor que puede implicar para la evaluación de la calidad de la atención en la dimensión interpersonal.

\section{Metodología}

Se propone una revisión de carácter exploratorio, se utilizaron bases de datos como Scielo, Medline, Redalyc, BVS y CINHAL. Los descriptores DeSC/ MeSH utilizados para realizar la búsqueda tanto en el idioma inglés como español fueron: "comunicación en salud”, "relación enfermera paciente”, “cuidado de enfermería”, "psicometría/enfermería”. La búsqueda permitió localizar publicaciones de los últimos 15 años (2002-2016), y algunas fuera de este periodo, pero que dan sustento al presente trabajo. Dichas publicaciones proceden del continente americano y europeo. Se revisaron 76 artículos, 26 pertenecen al método cualitativo, 45 aplican metodologías cuantitativas y cinco se abocan a la revisión de la literatura.

Se concentraron los datos en una tabla que muestra los aspectos principales de cada artículo: referencia hemerográfica, población, nivel de atención, servicio, elementos de la comunicación que se evaluaron, los cuales permitieron la operacionalización del presente trabajo.

Conforme al objetivo de este trabajo se establecieron los siguientes criterios: los artículos tenían que estar en texto completo, en el idioma español, inglés o portugués, utilizar una metodología cuantitativa y explorar a población de adultos, además el contenido del trabajo debería abordar temas sobre diseño, validación y/o aplicación de instrumentos que miden la comunicación o la relación entre el personal de enfermería y el paciente. Se incluyeron artículos originales y de revisión concernientes con el tema.

Bajo estos criterios quedaron 25 artículos por analizar, que corresponden a la atención brindada en centros de salud, hospitalización en urgencias, unidad de cuidados intensivos y medicina interna. De estos, nueve hacen referencia a la aplicación de instrumentos, 10 a la validez y adaptación de instrumentos y seis al diseño y validación de instrumentos. En 18 artículos los pacientes evalúan el cuidado, en cinco es el personal de enfermería, en el resto la valoración se hace entre estudiantes/pacientes y personal de enfermería.

Con base en los contenidos identificados en los artículos revisados y en la propuesta del objetivo, se establecen tres grupos: 1) aplicación y validación, 2) diseño y validación, 3) adaptación y validación de instrumentos (Tabla 1).

\section{Resultados}

Primer grupo: Aplicación y validación

En este primer grupo se encuentran artículos en los que se utilizan instrumentos con pequeños ajustes a la versión original ${ }^{6,10,15}$, traducidos ${ }^{12}$, o aplicados en su versión original ${ }^{13}$. Entre los instrumentos originales se citaron: el PSI referido por Contreras ${ }^{12}$, el SERVQHOS citado por Borre ${ }^{15}$ y Mira $^{34}$, el LOPSS mencionado por García Juárez ${ }^{14}$, el Care Q y GATHA, citado por Landman ${ }^{11}$ y el EMES utilizado por Marín ${ }^{13}$.

En siete artículos los pacientes son quienes evaluaron su relación con el personal de enfermería por medio de instrumentos adaptados ${ }^{10-15,36,37}$; en otros dos los estudiantes de enfermería son quienes valoraron su capacidad para ser empáticos con los pacientes ${ }^{6}$ así como las habilidades sociales que posee el profesional de enfermería en el desarrollo de su práctica ${ }^{13}$, en este caso se tiene como antecedente de este estudio la aplicación del mismo instrumento, pero en una población de estudiantes de Medicina ${ }^{38}$.

Los instrumentos se aplicaron en su mayoría en instituciones de salud, tanto en el ámbito de hospitalización como en la consulta externa, en algunos casos se realiza la encuesta a los pacientes durante el tiempo en que se encontraban internados ${ }^{14} y$ en otros después de su egreso ${ }^{15,36}$. Los instrumentos que fueron aplicados cuentan con validación de contenido, de constructo, o convergente, solo en un artículo se menciona que se ocuparon todas estas modalidades, simultáneamente ${ }^{6}$. 
Tabla 1.Características de los artículos seleccionados

\begin{tabular}{|c|c|c|c|c|}
\hline \multicolumn{5}{|c|}{ Primer grupo: aplicación y validación } \\
\hline Referencia & Población & $\begin{array}{l}\text { Nivel } \\
\text { de atención }\end{array}$ & Servicio & $\begin{array}{c}\text { Elementos de } \\
\text { comunicación evaluados }\end{array}$ \\
\hline $\begin{array}{l}\text { Díaz-Narváez } \\
\text { VP et al }{ }^{6} \text {. }\end{array}$ & $\begin{array}{l}\text { Estudiantes Adultos } \\
\text { mayores de } 18 \text { años }\end{array}$ & $\begin{array}{l}\text { Primero a quinto } \\
\text { año de la carrera }\end{array}$ & No especificado & Empatía \\
\hline Dios Guerra $\mathrm{C}$ et $\mathrm{al}^{10}$. & $\begin{array}{l}\text { Pacientes Adultos } \\
\text { entre } 18 \text { y } 89 \text { años }\end{array}$ & Primer nivel & Sala de curas & $\begin{array}{l}\text { Trato digno, Empatía, Escucha activa, } \\
\text { Amabilidad, Comprensión }\end{array}$ \\
\hline $\begin{array}{l}\text { Landman Navarro } \\
\text { C et } \mathrm{al}^{11} \text {. }\end{array}$ & $\begin{array}{l}\text { Pacientes Adultos } \\
\text { mayores de } 18 \text { años }\end{array}$ & Segundo nivel & Unidad Médico Quirúrgica & $\begin{array}{l}\text { Comunicación verbal, } \\
\text { Comunicación no verbal } \\
\text { Apoyo emocional, Respeto } \\
\text { Empatía, Comprensión }\end{array}$ \\
\hline Contreras $\mathrm{R}$ et $\mathrm{al}^{12}$. & $\begin{array}{l}\text { Pacientes Adultos } \\
\text { mayores de } 18 \text { años }\end{array}$ & Segundo nivel & $\begin{array}{l}\text { Medicina Interna } \\
\text { Cirugía General }\end{array}$ & $\begin{array}{l}\text { Relación interpersonal enfermera-paciente } \\
\text { Comprensión, Amabilidad }\end{array}$ \\
\hline $\begin{array}{l}\text { Marín-Sánchez M, } \\
\text { León-Rubio } \mathrm{JM}^{13} \text {. }\end{array}$ & Estudiantes & No especificado & No especificado & $\begin{array}{l}\text { Habilidades sociales } \\
\text { Relación interpersonal }\end{array}$ \\
\hline $\begin{array}{l}\text { García-Juárez MR } \\
\text { et } \mathrm{al}^{14} \text {. }\end{array}$ & $\begin{array}{l}\text { Pacientes Adultos } \\
\text { mayores de } 18 \text { años }\end{array}$ & No especificado & $\begin{array}{l}\text { Unidad de Medicina Interna, } \\
\text { de Neurología y Rehabilitación }\end{array}$ & $\begin{array}{l}\text { Confianza } \\
\text { Comprensión, Amabilidad }\end{array}$ \\
\hline Borré-Ortiz Y et al ${ }^{15}$. & Pacientes & No especificado & No especificado & Amabilidad, Trato \\
\hline Siamian $\mathrm{H}$ et $\mathrm{al}^{36}$. & Profesionales de la salud & No especificado & Consulta externa & Trato, Comunicación \\
\hline $\begin{array}{l}\text { Rojas-Martínez W, } \\
\text { Barajas-Lizarazo } \mathrm{MA}^{37} \text {. }\end{array}$ & Pacientes & Primer nivel & Urgencias & Conforta, confianza \\
\hline \multicolumn{5}{|c|}{ Segundo grupo: diseño y validación } \\
\hline Referencia & Población & $\begin{array}{c}\text { Nivel } \\
\text { de atención }\end{array}$ & Servicio & $\begin{array}{c}\text { Elementos de } \\
\text { comunicación evaluados }\end{array}$ \\
\hline Leal-Costa $\mathrm{C}$ et $\mathrm{al}^{7}$. & Profesionales de la salud & No especificado & No especificado & $\begin{array}{l}\text { Comunicación, Empatía, } \\
\text { Respeto, escucha activa } \\
\text { autenticidad }\end{array}$ \\
\hline $\begin{array}{l}\text { Lagunes-Córdoba R, } \\
\text { Hernández- } \\
\text { Manzanares } \mathrm{M}^{21} \text {. }\end{array}$ & Pacientes & HRAE Veracruz & $\begin{array}{l}\text { Ginecología, Medicina } \\
\text { Interna, Traumatología }\end{array}$ & $\begin{array}{l}\text { Comunicación, Empatía } \\
\text { Autenticidad, Ayuda }\end{array}$ \\
\hline $\begin{array}{l}\text { Puebla-Viera DC, } \\
\text { Ramírez-Gutiérrez A². }\end{array}$ & $\begin{array}{l}\text { Pacientes entre } 20 \text { y } \\
50 \text { años de edad. }\end{array}$ & Primer nivel & $\begin{array}{l}\text { Medicina preventiva } \\
\text { Consulta externa }\end{array}$ & $\begin{array}{l}\text { Respeto } \\
\text { Trato digno }\end{array}$ \\
\hline $\begin{array}{l}\text { Parrado-Lozano } \\
\text { YM et } \mathrm{al}^{23} \text {. }\end{array}$ & $\begin{array}{l}\text { Pacientes Adultos } \\
\text { mayores de } 18 \text { años }\end{array}$ & No especificado & $\mathrm{UCI}$ & $\begin{array}{l}\text { Trato digno, Respeto, Empatía, } \\
\text { Estrategias comunicativas }\end{array}$ \\
\hline Müggenburg $\mathrm{C}$ et $\mathrm{a}^{24}$. & $\begin{array}{l}\text { Pacientes Adultos } \\
\text { mayores de } 18 \text { años }\end{array}$ & Tercer nivel & No especificado & $\begin{array}{l}\text { Empatía } \\
\text { Respeto }\end{array}$ \\
\hline Duffy JR et $\mathrm{al}^{25}$. & Pacientes & No especificado & $\begin{array}{l}\text { Ortopedia, Telemetría, } \\
\text { Unidades quirúrgicas }\end{array}$ & Respeto, Empatía, Ayuda \\
\hline \multicolumn{5}{|c|}{ Tercer grupo: diseño y validación } \\
\hline Referencia & Población & $\begin{array}{l}\text { Nivel } \\
\text { de atención }\end{array}$ & Servicio & $\begin{array}{c}\text { Elementos de } \\
\text { comunicación evaluados }\end{array}$ \\
\hline López-Santos V et al ${ }^{16}$. & $\begin{array}{l}\text { Pacientes Adultos } \\
\text { mayores de } 18 \text { años }\end{array}$ & Primer nivel & No especificado & $\begin{array}{l}\text { Habilidades técnicas } \\
\text { comunicativas }\end{array}$ \\
\hline $\begin{array}{l}\text { González- } \\
\text { Hernández } \mathrm{OJ}^{26} \text {. }\end{array}$ & $\begin{array}{l}\text { Pacientes Adultos } \\
\text { mayores de } 18 \text { años }\end{array}$ & No especificado & No especificado & $\begin{array}{l}\text { Apertura a la comunicación } \\
\text { Actitud }\end{array}$ \\
\hline Newman A, Helft P ${ }^{27}$. & Enfermeras & No especificado & Oncología & Comunicación \\
\hline Parra DI, Arango GL ${ }^{28}$. & $\begin{array}{l}\text { Enfermeras Adultos } \\
\text { mayores de } 18 \text { años }\end{array}$ & $\begin{array}{l}\text { Tercero y cuarto } \\
\text { nivel de atención }\end{array}$ & No especificado & Comunicación \\
\hline Morales-Castillo FA $^{29}$. & $\begin{array}{l}\text { Enfermeras Adultos } \\
\text { entre } 25 \text { y } 55 \text { años }\end{array}$ & Segundo nivel & $\begin{array}{l}\text { Medicina Interna, Cirugía } \\
\text { General y Ginecobstetricia }\end{array}$ & $\begin{array}{l}\text { Ayuda, Confianza } \\
\text { Expresar sentimientos }\end{array}$ \\
\hline González-Ortega Y30. & Pacientes & Tercer nivel & $\begin{array}{l}\text { Ortopedia, Neurocirugía } \\
\text { Urgencias, Cirugía General } \\
\text { Hematología }\end{array}$ & $\begin{array}{l}\text { Apoyo emocional } \\
\text { Interacción familia } \\
\text { Interacción paciente }\end{array}$ \\
\hline $\begin{array}{l}\text { Poblete-Troncoso } \\
\mathrm{MC}^{31}\end{array}$ & $\begin{array}{l}\text { Enfermeras Adultos } \\
\text { mayores de } 18 \text { años }\end{array}$ & No especificado & No especificado & Empatía, Comunicación \\
\hline Duffy JR et $\mathrm{al}^{32}$. & Pacientes & No especificado & No especificado & Comunicación, Ayuda \\
\hline Lange JW ${ }^{33}$. & Pacientes & No especificado & No especificado & $\begin{array}{l}\text { Amabilidad, Ayuda } \\
\text { Comunicación, Comprensión }\end{array}$ \\
\hline Mira JJ et $\mathrm{al}^{34}$. & Pacientes & No especificado & No especificado & $\begin{array}{l}\text { Confianza, Trato, Ayuda } \\
\text { Empatía }\end{array}$ \\
\hline $\begin{array}{l}\text { Barragán JA } \\
\text { Manrique-Abril FG }{ }^{35} \text {. }\end{array}$ & Pacientes & No especificado & Unidad Quirúrgica & $\begin{array}{l}\text { Confianza, Comprensión } \\
\text { Trato, Amabilidad }\end{array}$ \\
\hline
\end{tabular}


No todos los estudios presentan los instrumentos en los que se basaron, sólo en algunos casos aparecen la adaptación de éstos ${ }^{10,14}$, algunos autores muestran los ítems de los instrumentos en el apartado de resultados y hacen una comparación entre los porcentajes obtenidos durante su aplicación.

Los aspectos regularmente incluidos en los instrumentos, relacionados con una comunicación efectiva, son la empatía, las habilidades sociales y el apoyo emocional. Algunos autores además de evaluar la relación que existe entre el personal de enfermería y el paciente, investigan la calidad de la atención que se brinda en las instituciones hospitalarias ${ }^{14,37}$.

Aun, cuando todas las investigaciones abordan las relaciones interpersonales entre el profesional de enfermería y el paciente, algunas enfatizan en conceptos como: respeto ${ }^{11}$, empatia ${ }^{6,11}$, confianza ${ }^{11,37}$ amabilidad ${ }^{10,12,14}$ comprensión ${ }^{10-12,14}$ y tiempo en que ocurre la interacción ${ }^{10,12}$ (Tabla 1).

Dentro de las características analizadas en el desarrollo de los artículos concentrados en este grupo, se observó que algunos estudios reportan información sociodemográfica ${ }^{11,12}$, la mayoría de los estudios busca medir el grado de satisfacción de los pacientes respecto a aspectos técnicos y/o comunicativos con enfermería ${ }^{10}$, la competencia de enfermería en la comunicación ${ }^{11}$, la calidad en los cuidados brindados por enfermería ${ }^{15,37}$ la importancia de la empatía ${ }^{6}$ y el uso de habilidades sociales ${ }^{13}$.

Respecto a los hallazgos más importantes se pueden mencionar los siguientes: algunos trabajos identificaron un alto grado de satisfacción por parte de los pacientes con respecto a los cuidados brindados por el personal de enfermería, así como, por su capacidad comunicativa, sin embargo, los pacientes sugirieron que se les permitiera una mayor participación en la toma de decisión respecto a su cuidado ${ }^{10-12}$. En una de estas investigaciones se hizo la comparación entre los resultados obtenidos a través de la aplicación del instrumento con otros estudios similares ${ }^{12}$.

En cuanto a las conclusiones que establecieron los autores de los estudios revisados se citaron las siguientes: debe haber mejoras en el tiempo de atención a los pacientes, en las habilidades de comunicación y en los recursos de apoyo ${ }^{10}$; el estrés en enfermería afecta la calidad de la atención que se brinda a los pacientes; se recomienda dedicar más tiempo a la interacción enfermera(o)-paciente ${ }^{37}$; las mujeres muestran más insatisfacción en cuanto a la comunicación del personal de enfermería y el grado de satisfacción puede verse afectado cuando la persona permanece más días hospitalizada ${ }^{11}$.

Los instrumentos que se utilizaron en las investigaciones están conformados por preguntas cerradas ${ }^{6}$, 12-15, 37, otros más por preguntas abiertas y cerradas ${ }^{10,11}$, en dos casos no se especifican las características del instrumento utilizado como tipo de pregunta o escalas empleadas ${ }^{36}$. La escala tipo Likert fue la más utilizada, midió respuestas como satisfecho-insatisfecho ${ }^{10,11}$, totalmente de acuerdo-totalmente desacuerdo ${ }^{6,12,14}$, nada importante-muy importante ${ }^{37}$ y mucho peor de lo que esperaba-mucho mejor de lo que esperaba.

\section{Segundo grupo: Diseño y validación}

Los estudios de este grupo se basan en la construcción de los instrumentos y cuentan con validez de contenido, de constructo o con ambos, lo que favorece la calidad del contenido de cada reactivo ${ }^{7,21-25}$ Un instrumento de este grupo cuenta también con validez convergente, el instrumento fue aplicado en la misma población al que se le había dado otro formato equivalente, se obtuvieron resultados similares, lo que confirmó su validez convergente ${ }^{24}$.

Los elementos de la comunicación enfermera-paciente más considerados para esta valoración fueron: la empatía, el respeto, el trato digno y la escucha activa ${ }^{7,21,22,24}$. Solamente, en el caso de dos instrumentos se refiere de manera explícita la fundamentación de estos en el modelo de la teórica Jean Watson, en uno retoman cuatro factores, entre los que se encuentran la comunicación y la empatía ${ }^{21}$; en el otro no sólo se retoma a la teórica antes mencionada, también se tomaron como referentes a M. Leininger y a F. Nightingale, para fundamentar la creación del instrumento CAT $^{25}$ que mide la percepción del cuidado desde el punto de vista del paciente, este instrumento ha tenido una amplia trayectoria, ya que empezó con 130 reactivos, continúo con 100, para quedarse con 36 reactivos en una de las primeras etapas; la 
última versión cuenta con validez facial, de contenido y de constructo, quedó integrado por ocho factores o componentes del cuidado: 1) resolución mutua de problemas; 2) atención al consuelo o reconfortamiento; 3) respeto; 4) promoción del ánimo; 5) apreciación de significados únicos; 6) ambiente de sanación; 7) adhesión a las necesidades; 8) necesidades básicas humanas.

En otra investigación, que también menciona a las teóricas referidas en el párrafo anterior, se abocó a evaluar la percepción de los pacientes sobre el comportamiento de comunicación de enfermería a través de la creación del instrumento CECOP, el cual en un principio contaba con 23 reactivos pero después de pasar por un proceso de validez de constructo se redujo a 10 reactivos y dos factores, el cual reporta un KMO de 0.791 con una $\mathrm{p} \leq 0.001$, que indica la homogeneidad entre las variables de cada factor $\mathrm{y}$ heterogeneidad entre las variables de otros factores. El CECOP tiene varias similitudes con el instrumento CAT, ya que ambos se diseñaron para medir la interacción enfermera-paciente, en ambas investigaciones los pacientes calificaron positivamente la interacción con las enfermeras ${ }^{24,25}$. La aplicación de los instrumentos se dio en el ámbito hospitalario ${ }^{7,23,24}$, igual que en la mayoría de los estudios referidos en la categoría anterior y son los pacientes quienes evaluaron el comportamiento de las enfermeras.

En uno de los estudios seleccionados se emplearon dos instrumentos, en el primero el evaluador es el paciente y en el otro es el familiar quien evalúa, sin embargo el objetivo es el mismo, medir la relación interpersonal que existe con la enfermera en la $\mathrm{UCI}^{23}$.

La mayoría de los artículos no hacen referencia explícita sobre el marco teórico de enfermería que los sustenta, solamente un artículo describe ampliamente el fundamento para el desarrollo de su instrumento ${ }^{7}$.

En un artículo aparece el instrumento tal como fue diseñado ${ }^{21}$, en otro se muestra el primer instrumento que crearon, sujeto a validación de contenido, y el resultante de la validación de constructo, con reducción de reactivos ${ }^{24}$; en dos investigaciones aparecen sólo los ítems en las tablas de resultados ${ }^{7,25}$ y por último en dos investigaciones más, no se muestran ni los instrumentos ni los reactivos diseñados ${ }^{22,23}$.

Una investigación cuenta con datos socio demográficos ${ }^{24}$; la mayoría de los artículos abordan elementos de la comunicación como: el respeto ${ }^{7,22-25}$, la empatía ${ }^{7,21,23-25}$, el trato digno ${ }^{22,23}$, la autenticidad ${ }^{7,21}$, la expresión de ayuda ${ }^{21,25}$, la actitud de asistencia ${ }^{21,23,25}$, y las estrategias comunicativas ${ }^{23}$ (Tabla 1).

Se encontraron dos investigaciones que utilizaron el método Delphi durante su proceso de diseño ${ }^{7,23}$; en tres investigaciones hubo reducción de ítems, en el primer caso empezó con 15 y terminó con 13 reactivos ${ }^{21}$, en el segundo caso contaba con 23 y terminó con $10^{24}$ y en un último caso contaba con 100 y concluyó con 36 reactivos al término de este proceso ${ }^{25}$.

Lo que se pretendió observar en los diversos estudios fue la percepción que tienen los pacientes sobre la atención brindada por enfermería ${ }^{22}$, la conducta ética en enfermeria ${ }^{21}$, la relación interpersonal entre enfermera- paciente y enfermera-familiar ${ }^{22}$, o exclusivamente la interacción enfermera-paciente ${ }^{24,25}$.

\section{Tercer grupo: Adaptación y validación}

En este grupo se incorporan estudios que adaptaron instrumentos ya existentes, utilizados en diferentes poblaciones con diferentes culturas e idiomas ${ }^{31}$. La mayoría de estos instrumentos cuentan con validez facial, validez de contenido y/o validez de constructo, lo que favorece el sustento de los resultados. También, en un caso se analiza la validez convergente, se destaca la correlación positiva que existe entre los dos instrumentos que se utilizaron ${ }^{31}$.

En la mayoría de los casos, se hace referencia al proceso de traducción y re traducción del instrumento recomendado por las OMS, esto permite corroborar si el instrumento administrado en otra población tiene el mismo sentido que en su población de origen ${ }^{18,19}$.

Algunos instrumentos se caracterizan por tener varios años de trayectoria que los respalda, cuentan con versiones anteriores durante las cuales se aplicaron diferentes métodos de validación que les permitieron ir haciendo ajustes y adaptaciones, que pueden mejorar las mediciones de los constructos; el instrumento PCHE 3ra versión cuenta con una trayectoria de 10 años de construcción, pasó de tener 50 
reactivos a 32 reactivos, este instrumento está basado en la teoría del cuidado humano de J. Watson y la Teoría de la Enfermería como cuidado de Boykin y Schoenhofer ${ }^{33}$, el CAT también cuenta con una larga trayectoria durante la cual pasó por diversos ajustes durante su validación, contaba con 130 reactivos para terminar con 27, este instrumento también se basó en la teoría postulada por J. Watson ${ }^{32}$.

Otros dos estudios tienen como base teórica a Jean Watson y su teoría de cuidado transpersonal, retoman 10 factores considerados en la categoría anterior ${ }^{29}$, 31. En uno de los trabajos se integran los 10 factores en siete subescalas ${ }^{29}$. En el segundo trabajo se estudia la validez convergente entre CES y el $\mathrm{NCA}^{31}$, además se analiza en forma simultánea la validez divergente con el apoyo de la subescala de malestar personal, que mide la falta de empatía.

\section{Conclusiones}

Los hallazgos encontrados a partir de esta revisión conforme al objetivo planteado de identificar instrumentos que miden la comunicación enfermera paciente, así como su adaptación y aplicación en el área de enfermería, permiten advertir qué instrumentos se han utilizado en diferentes países a lo largo del tiempo, los cuales han experimentado ajustes y modificaciones; también se pudo apreciar que el personal de enfermería, centrado en sus valores profesionales, se ha planteado diseñar, adaptar y aplicar instrumentos que les ayuden a evaluar la calidad de la atención brindada desde el ángulo del usuario. Destacan aspectos como el respeto, la empatía, la confianza, la comprensión y la amabilidad.

Se puede deducir que el tema de comunicación enfermera paciente ha sido, ampliamente estudiado por la importancia que este tiene para la profesión de enfermería, centrado en el cuidado de la persona, mismo que requiere de un eje articulador como es la comunicación que se lleva a cabo entre el personal de enfermería y su paciente.

Sin embargo, aún existen retos relacionados con esta medición, dado que los resultados alcanzados pueden generar nuevas estrategias para mejorar esta comunicación en la práctica diaria de enfermería, sin dejar de reconocer que los instrumentos arrojan mediciones indirectas de fenómenos psicológicos, con las implicaciones éticas que conllevan. El seguir trabajando en este tema, implica la realización de adecuaciones a una realidad concreta, con el fin de lograr diagnósticos específicos y proponer alternativas viables particulares.

\section{Responsabilidades éticas}

Protección de personas y animales. Los autores declaran que para esta investigación no se han realizado experimentos en seres humanos ni en animales, ya que se trata de una investigación documental.

Confidencialidad de los datos. Los autores declaran que en este artículo no aparecen datos de pacientes. Financiamiento. Ninguno

Conflicto de intereses. Los autores declaran no tener conflicto de intereses.

\section{Referencias}

1. Müggenburg-Rodríguez Vigil MC, Riveros-Rosas A. Interacción enfermera-paciente y su repercusión en el cuidado hospitalario: Parte I. Enferm. univ. 2012; 9(1): 36-44.

https://doi.org/10.22201/eneo.23958421e.2012.1.244

2. Rocha-Oliveira T, Faria-Simões SM. La comunicación enfermera-cliente en el cuidado en las unidades de urgencias 24h: una interpretación en Travelbee. Enfermería glob. 2013; 12(30): 76-90.

https://doi.org/10.6018/eglobal.12.2.154941

3. Tazón-Ansola MP, García-Campayo J, Aseguinolaza-Chopitea L. Relación y comunicación. Madrid: Difusión Avances en Enfermería; 2000.

4. Landete-Belda L. La comunicación, pieza clave en enfermería. Enferm. Dermatol. 2012; 6(16): 16-9. http://bit.ly/2HCxFvw 
5. CormierWH, Cormier LS. Estrategias de entrevista para terapeutas: habilidades básicas e intervenciones cognitivo-conductuales. $3^{\mathrm{a}}$ ed. Bilbao: Desclée de Brouwer; 2000.

6. Díaz-Narváez VP, Muñoz-Gámbaro G, Duarte-Gómez N, Reyes-Martínez MC, Caro SE, CalzadillaNuñez A, et al. Empatía en estudiantes de enfermería de la Universidad Mayor, sede Temuco, IX región, Chile. Aquichan. 2014; 14(3): 388-402. https://www.doi.org/10.5294/aqui.2014.14.3.9

7. Leal-Costa C,Tirado-González S, Van-der Hofstadt Román CJ, Rodríguez-Marín J. Creación de la Escala sobre Habilidades de comunicación en Profesionales de la Salud, EHC-PS. An. psicol. 2016; 32(1): 49-59. http://dx.doi.org/10.6018/analesps.31.3.184701

8. Happ MB, Garrett K, DiVirgilio-Thomas D, Tate J, George E, Houze M, et al. Nurse-patient communication interactions in the intensive care unit. Am J Crit Care. 2011; 20(2): e28-e40. http://doi.org/10.4037/ajcc2011433

9. Devos-Barlem ELD, Portella do Nascimento-Rosenhein DPN, Lerch-Lunardi VL, Lunardi-Filho W. Comunicação como instrumento de humanização do cuidado de enfermagem: experiências em unidade de terapia intensiva. Rev. Eletrônica Enferm. 2008; 10 (4): 1041-9. https://bit.ly/2Hz3eWS

10. Dios-Guerra C, Alba-Dios MA, Ruiz-Moral R, Jiménez-García C, Pérula-de Torres LA, RodríguezBorrego MA.Valoración de la satisfacción de usuarios de consulta de enfermería en centros de salud a partir de indicadores de calidad técnicos y de comunicación. Enfermería glob. 2013; 12(3): 162-76. https://doi.org/10.6018/eglobal.12.3.153131

11. Landman-Navarro C, Cruz-Osorio MJ, García-García E, Pérez-Meza P, Sandoval-Barrera P, Serey-Burgos $\mathrm{K}$, et al. Satisfacción usuaria respecto a competencia de comunicación del profesional de enfermería. Cienc. enferm. 2015; 21(1): 91-102. http://dx.doi.org/10.4067/S0717-95532015000100009

12. Contreras-Nuñez R, Coronado-Martínez M, López-Villanueva NH, Aguilar-Hernández RM, Félix Alemán A, Vega-Alanís MC. Satisfacción con el cuidado de Enfermería del adulto mayor hospitalizado. Enferm. univ. 2008; 5(1): 14-20. https://doi.org/10.22201/eneo.23958421e.2008.1.439

13. Marín-Sánchez M, León-Rubio JM. Entrenamiento en habilidades sociales: un método de enseñanza-aprendizaje para desarrollar las habilidades de comunicación interpersonal en el área de enfermería. Psicothema. 2001; 13(2): 247-51. http://bit.ly/30yGGyg

14. García-Juárez MR, López-Alonso SR, Orozco-Cózar MJ, Caro-Quesada R, Ramos-Osquet G, MárquezBorrego MJ. Personalización enfermera y calidad percibida del cuidado en el ámbito hospitalario. Gac. sanit. 2011; 25(6): 474-82. https://doi.org/10.1016/j.gaceta.2011.04.010

15. Borré-Ortiz YM, Lenis-Victoria CA, González-Ruíz GE. Utilidad del cuestionario SERVQHOS-E para medir calidad percibida de la atención de enfermería. CES Salud Pública, 2014; 5(2), 127-36. http://bit.ly/2QbCccc

16. López-Santos V, Sánchez-Ramos JL, Toronjo-Gómez Á, Pedregal-González M, Rojas-Ocaña MJ, Contreras-Martín Á. Valoración del componente comunicativo/relacional de la práctica enfermera en Atención Primaria: El cuestionario GATHA-ENFERMERÍA. Index enferm. 2008; 17(3): 173-7. https://bit.ly/30mvuVq

17. Mestre-Escrivá V, Frías-Navarro MD, Samper-García P. La medida de la empatía: análisis del Interpersonal Reactivity Index. Psicothema. 2004; 16(2): 255-60. https://bit.ly/2Jv2kyr

18. González-Ortega Y. La enfermera experta y las relaciones interpersonales. Aquichan. 2007; 7 (2):1308. http://bit.ly/2WWZWDA

19. Madrigal-Ramírez MC, Forero-Pulido C, Escobar CL. La comunicación, piedra angular en el cuidado de enfermería. Investig. enferm. imagen desarro. 2013; 15(2): 49-63. https://bit.ly/2VOuVoY

20. Maqueda-Martínez MÁ, Martín-Ibañez L. La habilidad de comunicar: caminando hacia el paciente. Rev. esp. comun. salud. 2012; 3(2): 158-66. http://bit.ly/2Wmmjp2

21. Lagunes-Córdoba R, Hernández-Manzanares MA. Escala de evaluación de la conducta ética del personal de enfermería en el cuidado de los pacientes. Aquichan. 2012; 12(3): 252-62. http://bit.ly/30yxhXJ 
22. Puebla-Viera DC, Ramírez-Gutiérrez A, Ramos-Pichardo P, Moreno-Gómez MT. Percepción del paciente de la atención otorgada por el personal de enfermería. Rev. enferm. Inst. Mex. Seguro Soc. 2009; 17 (2): 97-102. http://bit.ly/2VEUrYy

23. Parrado-Lozano YM, Sáenz-Montoya XS, Soto-Lesmes V, Guáqueta-Parada SR, Amaya-Rey P, CaroCastillo CV, et al. Validez de dos instrumentos para medir la relación interpersonal de la enfermera con el paciente y su familia en la unidad de cuidado intensivo. Investig. enferm. imagen desarro. 2016; 18(1): 115-28. http://dx.doi.org/10.11144/Javeriana.ie18-1.vimr

24. Müggenburg C, Robles R, Valencia A, Hernández-Guillén MC, Olvera S, Riveros-Rosas. Evaluación de la percepción de pacientes sobre el comportamiento de comunicación del personal de enfermería: diseño y validación en población mexicana. Salud Ment. 2015; 38(4): 273-80. http://dx.doi.org/10.17711/SM.0185-3325.2015.037

25. Duffy JR, Hoskins L, Seifert RF. Dimensions of caring: psychometric evaluation of the caring assessment tool. ANS Adv Nurs Sci. 2007; 30(3): 235-45. https://doi.org/10.1097/01.ANS.0000286622.84763.a9

26. González-Hernández OJ. Validez y confiabilidad del instrumento. "Percepción de comportamientos de cuidado humanizado de enfermería PCHE $3^{a}$ versión”. Aquichan. 2015; 15(3): 381-92. http://dx.doi.org/10.5294/aqui.2015.15.3.6

27. Newman AR, Helft PR. Reliability and validity of a tool to assess oncology nurses' experiences with prognosis-related communication. Oncol Nurs Forum. 2015; 42(1): 64-73. https://doi.org/10.1188/15.ONF.64-73

28. Parra DI, Arango-Bayer GL.Validez y confiabilidad de las escalas de comunicación y coordinación para medir rol interdependiente en enfermería. Av. enferm. 2010; 28(1): 51-62. http://bit.ly/2LWj2J2

29. Morales-Castillo FA, Hernández-Cruz MC, Morales-Rodríguez MC, Landeros-Olvera EA. Validación y estandarización del instrumento: Evaluación de los comportamientos de cuidado otorgado en enfermeras mexicanas. Enferm. univ. 2016; 13(1): 3-11.

http://dx.doi.org/10.1016/j.reu.2015.11.005

30. González-Ortega Y. Instrumento Cuidado de comportamiento profesional: validez y confiabilidad. Aquichan 2008; 8(2): 170-82. http://bit.ly/2JtN9VZ

31. Poblete-Troncoso MC, Valenzuela-Suazo SV, Merino JM. Validación de dos escalas utilizadas en la medición del cuidado humano transpersonal basadas en la Teoría de Jean Watson. Aquichan 2012; 12(1): 8-21. https://bit.ly/2WUeRy6

32. Duffy JR, Brewer BB, Weaver MT. Revision and psychometric properties of the caring assessment tool. Clin Nurs Res. 2014; 23(1): 80-93. https://doi.org/10.1177/1054773810369827

33. Lange JW. Testing equivalence of Spanish and English versions: The LaMonica-Oberst (revised) patient satisfaction with nursing care scale. Res Nurs Health. 2002; 25(6): 438-51. https://doi.org/10.1002/nur. 10057

34. Mira JJ, Aranaz J, Rodríguez-Marín J, Buil MC, Vitaller J. SERVQHOS: un cuestionario para evaluar la calidad percibida de la atención hospitalaria. Med. prev. 1998; 4: 12-8. http://bit.ly/2HDz059

35. Barragán-Becerra JA, Manrique-Abril FG. Validez y confiabilidad del SERVQHOS para enfermería en Boyacá, Colombia. Av. enferm. 2010; 28(2): 48-61. https://bit.ly/2VADCxV

36. Siamian H, Bagheri-Nesami M, Darvish-Nia, R, Reza-Nezhad F, Akbari H, Balaghafari A, et al. Assessment of Interpersonal communication skills among Sari Health Centers' Staff. Mater Sociomed. 2014; 26(5), 324-8. http://doi.org/10.5455/msm.2014.26.324-328

37. Rojas-MartínezW, Barajas-Lizarazo MA.Percepción de la calidad del cuidado de enfermería en el servicio de urgencias. Rev. cienc. cuidad. 2012; 9(1): 13-23. https://doi.org/10.22463/17949831.448

38. Alcorta-Garza A, González-Guerrero JF, Tavitas-Herrera SE, Rodríguez-Lara FJ, Hojat M. Validación de la Escala de Empatía Médica de Jefferson en estudiantes de medicina mexicanos. Salud Ment. 2005; 28(5): 57-63. https://bit.ly/2EjrrA2 